\title{
Synthesis and characterization of photo-crosslinked poly(carbonate anhydrides)
}

\author{
B. van Bochove, J. V. Seppälä* \\ Polymer Technology, Department of Chemical and Metallurgical Engineering, School of Chemical Engineering, \\ Aalto University, 02150 Espoo, Finland
}

Received 13 September 2019; accepted in revised form 5 November 2019

\begin{abstract}
Biodegradable poly(carbonate anhydrides) based on poly(trimethylene carbonate) were synthesized by ring-opening polymerization. The hydroxyl-terminated oligomers were acid-functionalized with succinic anhydride and subsequently methacrylated to obtain photo-crosslinkable three-armed macromers with anhydride bonds. The degree of acid-functionalization was over $96 \%$, and the degree of methacrylation was over $80 \%$. The macromers were photo-crosslinked into networks at room temperature in a nitrogen atmosphere. In vitro degradation experiments in phosphate-buffered solution ( $\mathrm{pH} 7.4)$ at $37^{\circ} \mathrm{C}$ showed that the networks degraded rapidly with complete mass loss within $72 \mathrm{~h}$. For networks prepared from the higher molecular weight, the presence of an alkenyl chain in the anhydride bond resulted in lower water uptake and consequently in a decrease in the degradation rate. The degradation mechanism of these networks showed characteristics of surface erosion.
\end{abstract}

Keywords: biodegradable polymers, poly(trimethylene carbonate), poly(anhydrides), polymer degradation, polymer networks

\section{Introduction}

Biodegradable and biocompatible polymers receive significant interest in the biomedical field for applications such as implants materials and drug delivery devices. Poly(trimethylene carbonate) (PTMC) is an amorphous polymer which has a low glass transition temperature of approximately $-16^{\circ} \mathrm{C}$ [1]. The mechanical properties of PTMC depend strongly on the molecular weight of the polymer [2]. Linear, noncrosslinked high molecular weight PTMC is a tough and flexible polymer that degrades via surface erosion in vivo without the release of acidic compounds $[3,4]$. Due to its biocompatibility and biodegradability PTMC has potential for biomedical applications [5-12]. Non-crosslinked low molecular weight PTMC is soft and gummy with a low modulus and tensile strength. However, by photo-crosslinking methacrylate-functionalized low molecular weight
PTMC oligomers (macromers), networks that are creep resistant and have excellent mechanical properties can be prepared [13]. Due to these properties PTMC-based networks have been investigated for application as meniscus implants [14], microvascular networks [15], bone implants [16], intervertebral disk implants [17], and drug delivery devices [18]. By adjusting the molecular weight of the macromers used to prepare the networks, the mechanical properties can be tuned [13]. Additionally, the molecular weight of the macromers also influences the degradation rates of the networks [19]. In general, if the molecular weight of the macromers increases, so does the degradation rate. However, the rate of degradation is slow for networks prepared from macromers with molecular weights between 10 and $30 \mathrm{~kg} / \mathrm{mol}$, with a mass loss of only $0.5-3.7 \%$ in 36 weeks in vivo [19]. Crucially, the degradation appeared to take 
place via surface erosion. This allows the networks to obtain their mechanical strength during degradation, which is essential for load-bearing applications [1]. Previously, our group has reported on the preparation and characterization of photo-crosslinked poly(ester anhydrides) prepared from poly( $\varepsilon$-caprolactone) (PCL) and polylactide (PLA) [20-24]. Hydroxyl-terminated oligomers are acid-substituted with an anhydride compound to yield acid-terminated oligomers. The anhydride compound may be succinic anhydride or alkenyl succinic anhydrides containing 8-18 carbons in the alkenyl chain. Subsequent methacrylate-functionalization of these compounds results in crosslinkable macromers. Regular PCL networks, without anhydride bond, degrade very slowly $[25,26]$. The addition of a labile anhydride bond results in networks that degrade much faster $[21,22]$, in 48 hours for the networks prepared with succinic anhydride to 64 to 72 hours for anhydrides containing an alkenyl chain with 12 or 18 carbons respectively. Increasing the macromer molecular weight also decreases the degradation rate [24].

Poly(ester anhydrides) networks are promising networks for drug delivery applications. While drug delivery with hydrophobic networks such as PTMC networks is possible, due to lack of microporosity and water uptake, the mesh size of the densely crosslinked network is the limiting factor in the drug release [27]. It is expected that the presence of succinic anhydride in the PTMC networks increases the water uptake and accelerates the degradation rate resulting in drug release with the polymer swelling and degradation as driving factors in the drug release. This would make PTMC-anhydride networks attractive materials for drug release applications. Also, developing PTMC-anhydride networks results in a material with tailorable degradation properties. Thus, the development of PTMC-anhydride networks is interesting not only for drug delivery but for the broader biomedical materials field in general.

Therefore, in this first study, a set of PTMC-anhydride networks with different succinic anhydrides was prepared and characterized by water uptake, degradation rate and type of erosion. To prepare these networks, low molecular weight macromers were chosen as (i) the degradation for those networks was expected to be faster, and (ii) the oligomer viscosity of these lower molecular weight macromers allows for similar synthesis without solvents.

\section{Experimental}

\subsection{Materials}

Trimethylene carbonate $(\geq 99.0 \%$, TMC) was purchased from ForYou Medical Co. (China). Trimethylol propane (TMP), succinic anhydrides, methacrylic anhydride, hydroquinone, tin(II) 2-ethylhexanoate $\left(\mathrm{Sn}(\mathrm{Oct})_{2}\right)$ and d-chlorofrom were obtained from Sigma-Aldrich (USA). Chlorofrom was obtained from Merck (Germany) and TPO-L (Ethyl Phenyl (2,4,6-trimethylbenzoyl)phosphinate) was obtained from Carbosynth Limited (UK). Phosphate-buffered solution (PBS, pH 7.4) was purchased from Oy FFChemicals $\mathrm{Ab}$ (Finland). All chemicals were used as received.

\subsection{Synthesis and characterization of PTMC-anhydride macromers}

Three-armed hydroxyl-terminated PTMC oligomers were prepared by a 3-day ring-opening polymerization of TMC at $130^{\circ} \mathrm{C}$ under nitrogen atmosphere using TMP as initiator and $\mathrm{Sn}(\mathrm{Oct})_{2}$ as catalyst. By adjusting the monomer to initiator ratio different molecular weights $\left(M_{\mathrm{n}}\right)$ could be obtained. For each molecular weight, two out of three oligomers were acidfunctionalized by a $3 \mathrm{~h}$ reaction of the hydroxyl groups with (alkenyl) succinic anhydride $(3.09 \mathrm{~mol} / \mathrm{mol}$ oligomer) at $140^{\circ} \mathrm{C}$. To obtain photo-crosslinkable macromers, all oligomers are finally methacrylatefunctionalized with methacrylic anhydride ( $7.5 \mathrm{~mol} / \mathrm{mol}$ oligomer) in a $24 \mathrm{~h}$ reaction at $60^{\circ} \mathrm{C}$ in the presence of hydroquinone $(0.1 \mathrm{wt} \%$ relative to the amount of monomer) preventing premature crosslinking. A reaction scheme of the preparation of photo-crosslinkable PTMC-anhydride macromers is shown in Figure 1.

The number average molar mass $\left(M_{\mathrm{n}}\right)$ and monomer conversion of the oligomers, the degree of acid substitution, and degree of methacrylate functionalization were determined with ${ }^{1} \mathrm{H}$ and ${ }^{13} \mathrm{C}$ NMR (Brüker NMR Spectrometer AV III 400).

The thermal properties of the macromers were determined by differential scanning calorimetry (DSC) using a TA Instruments MT-DSC Q2000. Samples weighing 5-10 $\mathrm{mg}$ were heated from -90 to $100^{\circ} \mathrm{C}$ at a rate of $10^{\circ} \mathrm{C} / \mathrm{min}$ and subsequently cooled to $-90^{\circ} \mathrm{C}$ at a rate of $10^{\circ} \mathrm{C} / \mathrm{min}$. After 5 minutes at $-90^{\circ} \mathrm{C}$ a second heating scan to $100^{\circ} \mathrm{C}$ at a rate of $10^{\circ} \mathrm{C} / \mathrm{min}$ was run, which was used to determine the glass transition temperatures $\left(T_{\mathrm{g}}\right)$ of the macromers. 


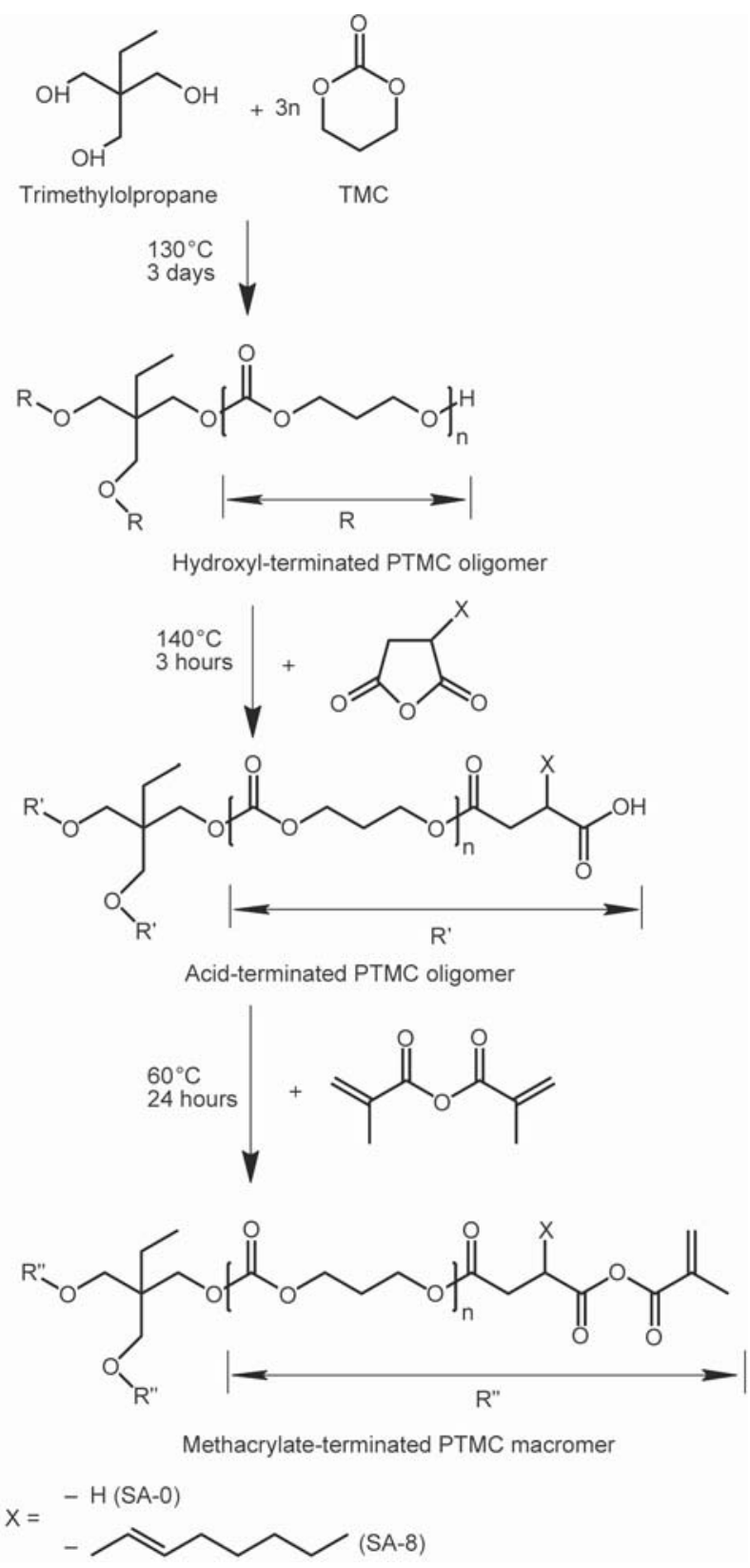

Figure 1. Reaction scheme of the preparation of photocrosslinkable PTMC-anhydride macromers.

\subsection{Network formation and characterization}

To obtain photo-crosslinked networks, the liquid macromers were mixed with $5 \mathrm{wt} \%$ TPO-L photoinitiator and poured into cylindrical molds $(d=$ $5 \mathrm{~mm}, h=2.5 \mathrm{~mm})$. The macromers were subsequently crosslinked at room temperature under nitrogen atmosphere in a self-made crosslink box for $30 \mathrm{~min}$ at $395-405 \mathrm{~nm}$ at an intensity of $1 \mathrm{~mW} / \mathrm{cm}^{2}$. The obtained networks were post-cured under visible light for 40 minutes and then stored in vacuo.

The volume degree of swelling $(q)$ and gel content of the obtained networks were determined in triplicate at room temperature by swelling the networks in chloroform for 24 hours. The values were calculated using Equations (1) and (2):

$q=1+\frac{m_{\text {swollen }}-m_{\mathrm{dry}}}{m_{\mathrm{dry}}} \cdot \frac{\rho_{\mathrm{p}}}{\rho_{\mathrm{s}}}$

Gel content $[\%]=\frac{m_{\mathrm{dry}}}{m_{\text {initial }}} \cdot 100$

where $m_{\text {swollen }}$ is the mass of the swollen networks, $m_{\text {dry }}$ the mass of the insoluble part of the networks after drying, $m_{\text {initial }}$ the initial mass of the networks, and $\rho_{\mathrm{p}}$ and $\rho_{\mathrm{s}}$ are the densities of PTMC $\left(1.31 \mathrm{~g} / \mathrm{cm}^{3}\right)$ and chloroform $\left(1.48 \mathrm{~g} / \mathrm{cm}^{3}\right)$, respectively.

The thermal properties of the networks were determined by DSC, equally as previously described for the macromers. In addition, the thermal decomposition temperatures of the polymer networks were determined by Thermogravimetric Analysis (TA Instruments TGA Q500). Samples were heated from 30 up to $600^{\circ} \mathrm{C}$ at $10^{\circ} \mathrm{C} / \mathrm{min}$ under a nitrogen atmosphere. The hydrolysis of the networks in PBS $(\mathrm{pH}=7.4)$ was performed by immersing the specimens in $10 \mathrm{~mL}$ $\left(37^{\circ} \mathrm{C}\right.$, shaking at $\left.100 \mathrm{rpm}\right)$ of PBS and recovery of the specimens at pre-determined intervals. For each time point, three parallel specimens were weighted and the thickness was measured before degradation. At recovery, the specimens were weighed again to determine the water-uptake. The specimens were then dried in vacuum until constant weight was achieved and the mass and thickness of the specimens could be determined. The water uptake was calculated using Equation (3):

Water uptake $[\%]=\frac{m_{\mathrm{wet}}-m_{\mathrm{dry}}}{m_{\mathrm{dry}}} \cdot 100$

where $m_{\text {wet }}$ is the mass of the specimens at the moment of recovery from the buffer and $m_{\text {dry }}$ is the mass of specimens after drying.

\section{Results and discussion}

\subsection{Polymer synthesis and macromer analysis}

The reaction scheme of the PTMC-anhydride polymerizations is shown in Figure 1. The synthesis of photo-crosslinkable PTMC-anhydride macromers consisted of three steps. First, three-armed hydroxylterminated oligomers were synthesized by ringopening polymerization of TMC using TMP as initiator. By adjusting the monomer to initiator ratio, the molecular weight of the oligomer could be controlled. The targeted molecular weights were 900 
and $2000 \mathrm{~g} / \mathrm{mol}$, hereafter called PTMC-900 and PTMC-2K, respectively. Second, the hydroxyl termination was converted into acid termination by a reaction with succinic anhydride or alkenyl succinic anhydride containing 8 carbons in the alkenyl chain. Third, the acid termination was changed to methacrylate termination by a reaction with methacrylic anhydride. The reaction conditions were derived from previous work on TMC syntheses and the preparation of poly(ester anhydrides) [13, 20, 28].

Table 1 gives an overview of the obtained oligomer molecular weights and monomer conversions, as determined by ${ }^{1} \mathrm{H}-\mathrm{NMR}$. By comparing the area of the $-\mathrm{CH}_{3}$ peak of the TMP initiator at $\delta 0.92 \mathrm{ppm}$ with the area of the PTMC methylene peak at $\delta 4.24 \mathrm{ppm}$ the $M_{\mathrm{n}}$ of the oligomer could be determined. Besides, by comparing the PTMC methylene peak with the $\mathrm{MC}$ monomer peak at $\delta 4.45 \mathrm{ppm}$ the conversion was calculated. The regular (non-acid-substituted) PTMC oligomers were directly methacrylate-functionalized. The degree of functionalization (DF) of these macromers was also determined by ${ }^{1} \mathrm{H}-\mathrm{NMR}$. Methacrylate-functionalization was confirmed by the appearance of the $\mathrm{H}$-signals of the $\mathrm{C}=\mathrm{CH}_{2}$ groups at $\delta 5.58 \mathrm{ppm}$ and $\delta 6.11 \mathrm{ppm}$. The degree of functionalization was calculated by comparing these peaks with the TMP initiator peak.

The acid functionalization of PTMC oligomers can be confirmed by $1 \mathrm{H}-\mathrm{NMR}$ by the disappearance of the signals of the last $\mathrm{CH} 2$ group of the oligomer chain end at $\delta 3.7 \mathrm{ppm}$, as was previously described for acid-substituted lactide and caprolactone polymers [20]. The degree of substitution (DS) was shown to $>96 \%$ in all cases.

The quantitative analyses of the methacrylate functionalization of acid substituted oligomers are easier to be done by ${ }^{13} \mathrm{C}$-NMR [20]. Besides, we calculated the oligomer molecular weight. Table 1 gives an overview of obtained results. To calculate the $M_{\mathrm{n}}$, we compared the signal of the carbon of the TMP initiator at $\delta 7.2 \mathrm{ppm}$ to the carbons of the repeating monomer unit at $\delta 28.0$ and $\delta 64.3 \mathrm{ppm}$.

The degree of methacrylate functionlalization was calculated by comparing the signal of the TMP initiator with the signal of the methacrylate that appears $\delta 163.1 \mathrm{ppm}$ after functionalization. The DF of all macromers was $>80 \%$. A complete overview of the results can be seen in Table 1, while Figure 2 shows the ${ }^{1} \mathrm{H}$ NMR spectra and Figure 3 the ${ }^{13} \mathrm{C}-\mathrm{NMR}$ spectra of typical PTMC-anhydrides. All obtained macromers were viscous liquids at room temperature.

The thermal properties of the obtained macromers were analyzed with DSC; an overview of the obtained $T_{\mathrm{g}}$ 's is shown in Table 1 . The observed $T_{\mathrm{g}}$ 's of the regular PTMC macromers are slightly lower than those previously reported of similar molecular weights [13], but this is likely due to remaining methacrylic anhydride and methacrylic acid. There does not appear to
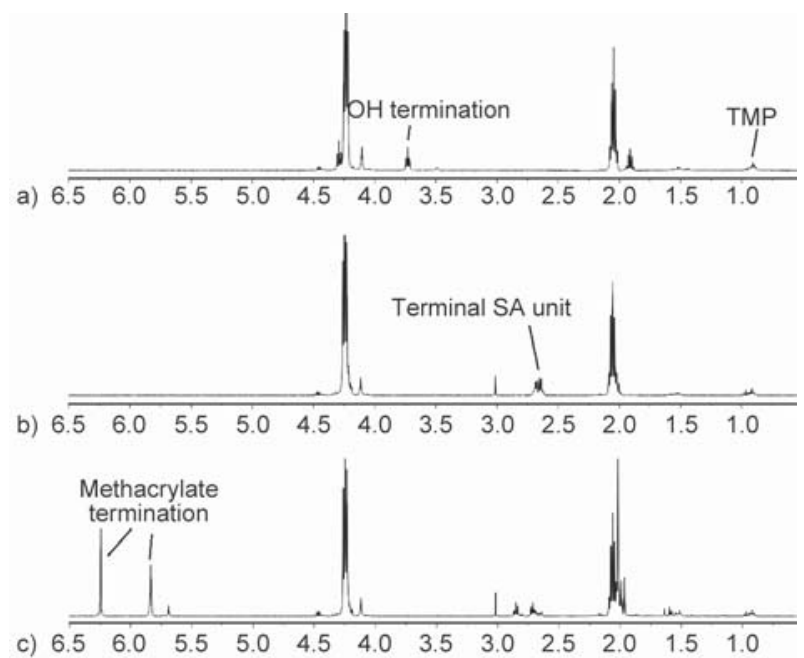

Figure 2. ${ }^{1} \mathrm{H}-\mathrm{NMR}$ spectra of (a) PTMC oligomer, (b) acidsubstituted PTMC, and (c) methacrylated PTMC anhydride.

Table 1. Properties of PTMC and PTMC-anhydride macromers as determined by ${ }^{1} \mathrm{H}-\mathrm{NMR},{ }^{13} \mathrm{C}-\mathrm{NMR}$ and DSC.

\begin{tabular}{|c|c|c|c|c|c|c|c|}
\hline \multirow[b]{2}{*}{ Macromer } & \multicolumn{2}{|c|}{$M_{\mathrm{n}}$} & \multirow{2}{*}{$\begin{array}{c}\text { Conv. } \\
{[\%]}\end{array}$} & \multirow{2}{*}{$\begin{array}{c}\text { DS } \\
{[\%]}\end{array}$} & \multirow{2}{*}{$\begin{array}{c}\text { DF } \\
{[\%]}\end{array}$} & \multirow[b]{2}{*}{$T_{\mathrm{g}}$ (onset) } & \multirow{2}{*}{$\begin{array}{c}T_{\mathrm{g}} \text { (mid-point) } \\
{\left[{ }^{\circ} \mathrm{C}\right]}\end{array}$} \\
\hline & $\begin{array}{c}{ }^{1} \mathrm{H}-\mathrm{NMR} \\
{[\mathrm{g} / \mathrm{mol}]}\end{array}$ & $\begin{array}{c}{ }^{13} \mathrm{C}-\mathrm{NMR} \\
{[\mathrm{g} / \mathrm{mol}]}\end{array}$ & & & & & \\
\hline PTMC-900 & 1000 & - & 99 & - & $79^{\mathrm{a}}$ & -83 & -82 \\
\hline PTMC-900-SA(0) & 1000 & 1000 & 99 & 100 & $92^{\mathrm{b}}$ & -79 & -74 \\
\hline PTMC-900-SA(8) & 1000 & 1000 & 99 & 100 & $99^{\mathrm{b}}$ & -80 & -77 \\
\hline PTMC-2K & 2200 & - & 98 & - & $80^{\mathrm{a}}$ & -69 & -62 \\
\hline PTMC-2K-SA(0) & 2100 & 2200 & 99 & 100 & $87^{\mathrm{b}}$ & -65 & -59 \\
\hline PTMC-2K-SA(8) & 2200 & 2100 & 99 & 96 & $83^{\mathrm{b}}$ & -71 & -64 \\
\hline
\end{tabular}

${ }^{\text {a }} \mathrm{DF}$ determined by ${ }^{1} \mathrm{H}-\mathrm{NMR}$

${ }^{\mathrm{b}} \mathrm{DF}$ determined by ${ }^{13} \mathrm{C}-\mathrm{NMR}$ 


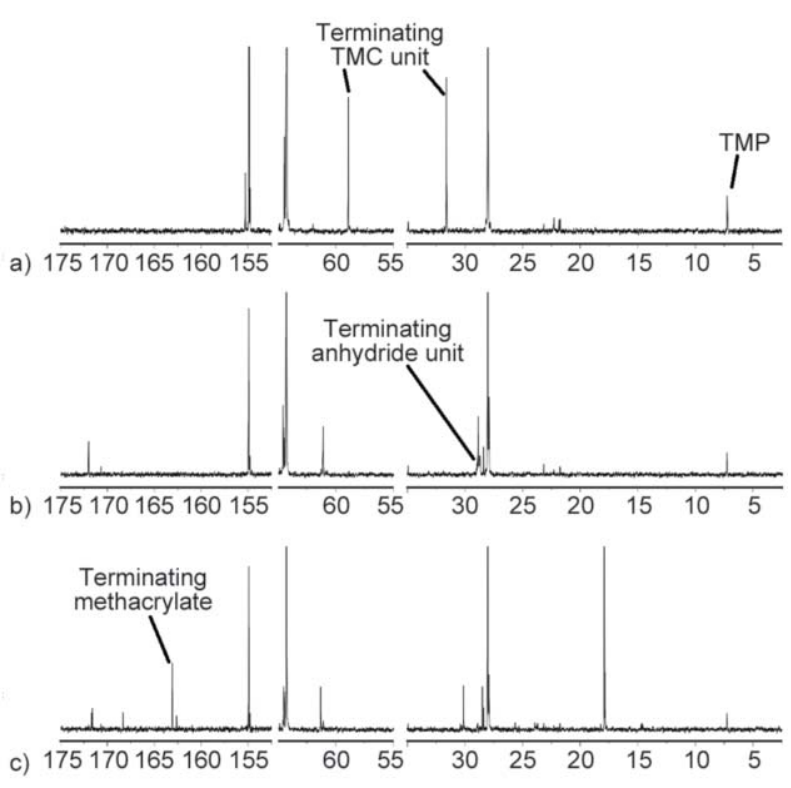

Figure 3. ${ }^{13} \mathrm{C}$-NMR spectra of (a) PTMC oligomer, (b) acidsubstituted PTMC, and (c) methacrylated PTMC anhydride.

be a significant effect of the incorporation of an anhydride group in the macromer on the $T_{\mathrm{g}}$.

\subsection{Network formation and characterization}

PTMC and PTMC-anhydride network cylinders were formed by photo-crosslinking the macromers using TPO-L as photo-initiator in a UV crosslinking box. After crosslinking, the cylinders were removed from the mold and characterized. An overview of the network properties is shown in Table 2. Network cylinders with gel contents between 92 and 100\% were prepared. It should be noted that in initial experiments with networks prepared from two-armed macromers, maximum gel contents were not higher than approximately $70 \%$ (data not shown).

The degree of swelling in chloroform increased with the addition of the succinic anhydride to the macromer molecule structure. This is especially the case for the networks prepared with the higher molecular weight macromers. During the swelling process, the networks prepared from the lower molecular weight macromers were prone to defragmentation due to the brittleness of these networks.

The glass transitions of the PTMC-2K and all three PTMC-900 networks could not be observed. This is likely due to the low molecular weight of the macromers, which results in densely crosslinked networks. As a result, the chain mobility of polymer chains in the networks is already strongly limited which means that the difference in mobility above and below the $T_{\mathrm{g}}$ is rather small and goes undetected. The $T_{\mathrm{g}}$ 's of PTMC-2K-SA(0) and PTMC-2K-SA(8) were close to the $T_{\mathrm{g}}$ of previously reported PTMC networks prepared from macromers with similar molecular weight [13]. The onset of the thermal decomposition temperatures of the polymer networks was between $267-301{ }^{\circ} \mathrm{C}$.

\subsection{Hydrolytic degradation of photo- crosslinked PTMC-anhydride networks}

The in vitro hydrolytic degradation of photo-crosslinked PTMC-anhydride networks was investigated in PBS (pH 7.4) at $37^{\circ} \mathrm{C}$ by determining the loss of mass and thickness of cylindrical samples over time. In addition, the water uptake of the samples was determined. Figure 4 shows the relative remaining mass and relative remaining thickness of the PTMC900 networks in Figure 4a and Figure 4b respectively. As has been shown previously, regular PTMC networks do not show mass loss under this condition for this period [19]. The PTMC-900-anhydride networks, on the other hand, do show degradation with $33.1 \pm 16$ and $43.3 \pm 8 \%$ average relative mass remaining after 48 hours for succinic anhydride and alkenyl succinic anhydride containing PTMC respectively. Besides, for both these samples a decrease in thickness is clearly noticeable. Further evidence of the decrease in thickness is shown in Figure 5. This indicates that these networks degrade via a surface erosion mechanism. Figure $4 \mathrm{c}$ shows that the mass is decreasing at a higher rate as compared to the thickness. If the degradation only proceeded from the top

Table 2. Properties of PTMC and PTMC-anhydride networks prepared by photo-crosslinking their respective macromers.

\begin{tabular}{|c|c|c|c|c|c|}
\hline Network & $\begin{array}{c}\text { Gel content } \\
{[\%]}\end{array}$ & Degree of swelling & $\begin{array}{c}T_{\mathrm{g}} \text { (onset) } \\
{\left[{ }^{\circ} \mathrm{C}\right]}\end{array}$ & $\begin{array}{c}T_{\mathrm{g}} \text { (mid-point) } \\
{\left[{ }^{\circ} \mathrm{C}\right]}\end{array}$ & $\begin{array}{c}T_{\text {decomposition }} \text { onset } \\
{\left[{ }^{\circ} \mathrm{C}\right]}\end{array}$ \\
\hline PTMC-900 & $100 \pm 0.1$ & $1.7 \pm 0.2$ & - & - & 293 \\
\hline PTMC-900-SA(0) & $100 \pm 0.1$ & $1.9 \pm 0.1$ & - & - & 280 \\
\hline PTMC-900-SA(8) & $93 \pm 5.8$ & $2.4 \pm 0.8$ & - & - & 301 \\
\hline PTMC-2K & $97 \pm 2.2$ & $2.2 \pm 0.1$ & - & - & 267 \\
\hline PTMC-2K-SA(0) & $99 \pm 2.0$ & $2.9 \pm 0.1$ & -11 & -6 & 285 \\
\hline PTMC-2K-SA(8) & $92 \pm 1.0$ & $2.8 \pm 0.1$ & -15 & -13 & 271 \\
\hline
\end{tabular}



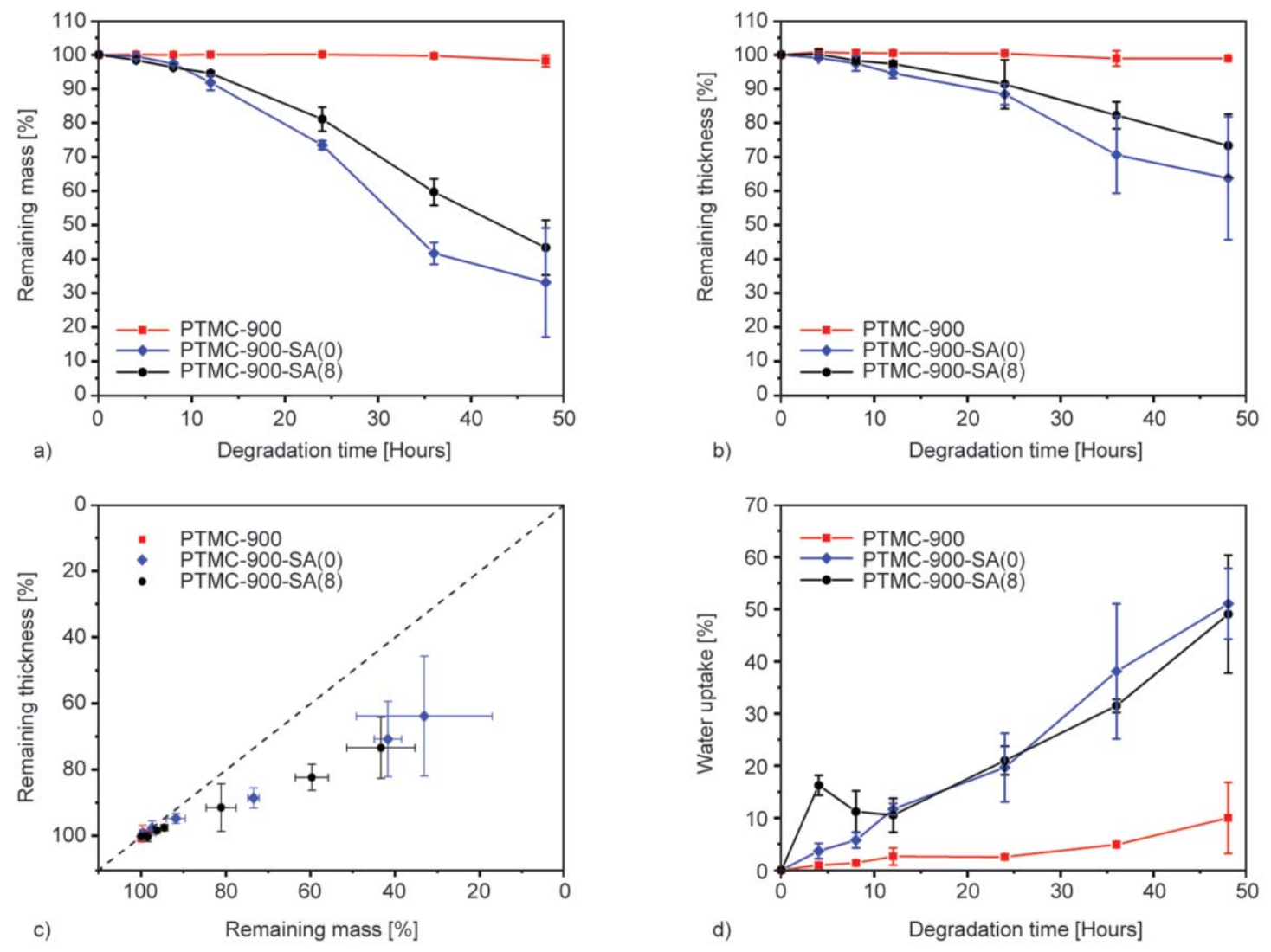

Figure 4. Degradation of PTMC-900-anhydride. (a) Relative remaining mass as a function of time. (b) Relative remaining thickness as a function of time. (c) Relative remaining thickness as a function of relative remaining mass. (d) Water update of the cylinders as a function of time.

and bottom of the cylinders this rate would be equal [29], and the points would be shown on the dotted line in Figure 4c. The difference in rate indicates that these networks also loose mass elsewhere, likely on the side of the networks. Figure $4 \mathrm{~d}$ shows that the water uptake of the PTMC-900-SA(0) is equal to that of PTMC-900-SA(8) which likely is the reason why the degradation of the PTMC-900-SA(8) has a similar degradation rate as PTMC-900-SA(0) while the additional alkenyl chain of the PTMC-900-SA(8) would be expected to significantly slow down the degradation rate [23].

Figures $6 \mathrm{a}$ and $6 \mathrm{~b}$ show the relative remaining mass and relative remaining thickness of the PTMC-2K networks. Like the regular PTMC-900 networks, the regular PTMC-2K networks do not degrade. The PTMC-2K-SA(0) networks do show significant mass loss and loss of thickness in $48 \mathrm{~h}$. As can be seen in Figure $6 c$, the decrease in mass is higher than the decrease in thickness, indicating that this network degrades similarly to the PTMC-900-SA(0) and PTMC-900-SA(8) networks. Figure 5 shows the loss of the thickness of $v s$. time. The PTMC-2K-SA(8) networks do show a small loss of mass and thickness after $48 \mathrm{~h}$ but not enough to determine the degradation mechanism. Figure $6 \mathrm{~d}$ shows that the water uptake of the PTMC-2K-SA(8) networks increases at a much slower rate than that of the PTMC-2K-SA $(0)$ networks. This may be the reason the degradation of PTMC-2K-SA(8) networks is slower.

In preliminary experiments, the degradation products of the PTMC-anhydride network degradation in PBS were collected, dried in vacuum at 40 degrees, and used for NMR analyses. The degradation products showed the characteristic TMC peaks at $\delta 2.05 \mathrm{ppm}$ and $\delta 4.25 \mathrm{ppm}$ as well as the succinic anhydride peaks at $\delta 2.65 \mathrm{ppm}$. However, there were no methacrylate peaks visible at $\delta 5.58 \mathrm{ppm}$ and $\delta$ $6.11 \mathrm{ppm}$ indicating that the degradation products are acid-functionalized PTMC oligomers.

Some limitations concerning the above-descibed experiments and obtained results need to be discussed. First, the degradation experiments were only $48 \mathrm{~h}$ long. Experiments with longer timepoints were attempted, but for the fastest degrading networks the next time point of 72 hours could not be completed 
a)

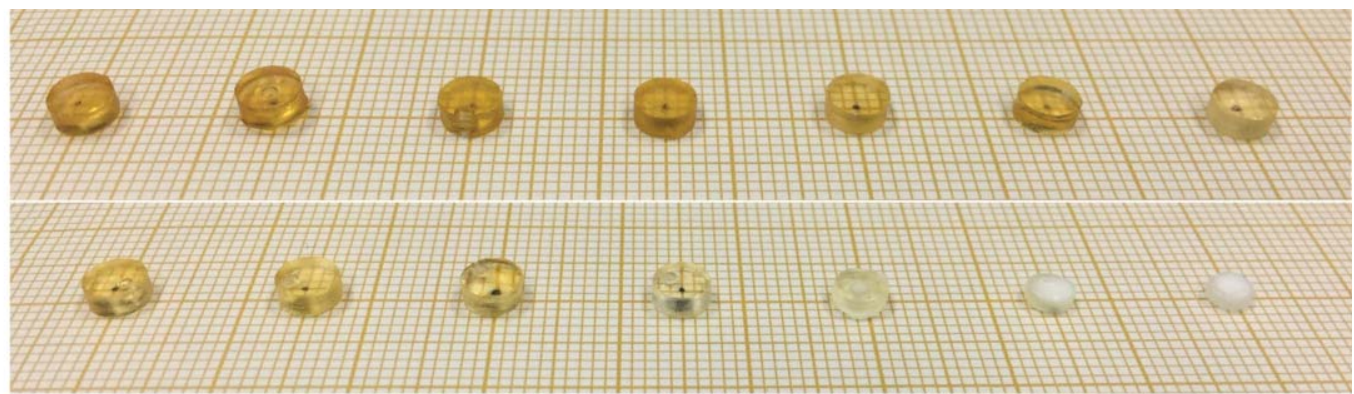

c)

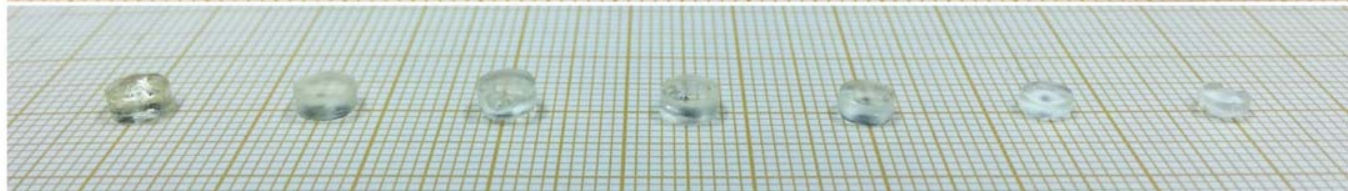

d)
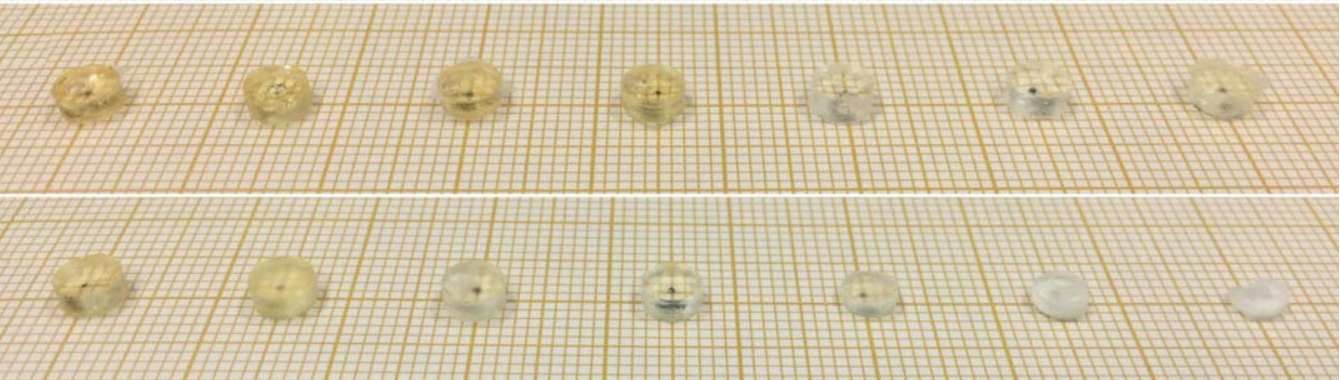

f)

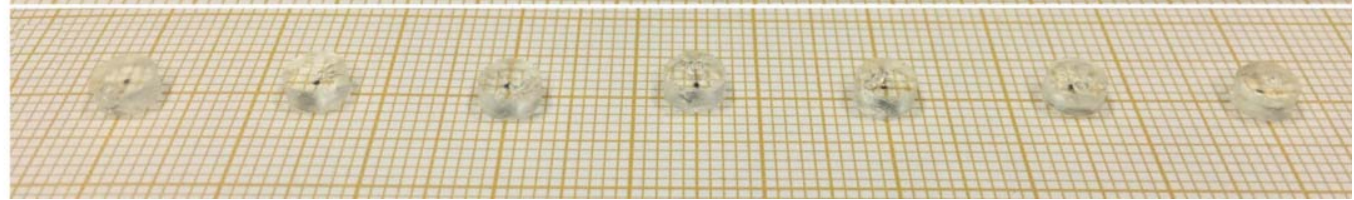

Figure 5. Pictures of dried PTMC-900 (a), PTMC-900-SA(0) (b), PTMC-900-SA(8) (c), PTMC-2K (d), PTMC-2KSA(0) (e), and PTMC-2K-SA(8) (f) networks exposed to PBS for (from left to right) 0, 4, 8, 12, 24, 36 and $48 \mathrm{~h}$.

due to the networks being completely degraded somewhere between 48 and $72 \mathrm{~h}$. Especially for networks prepared from PTMC-2K-SA(8) longer degradation times would be interesting to determine if the increase in water uptake will result in an increase in the rate of degradation. Longer degradation times can also be achieved by increasing the length of the alkenyl chains or the molecular weight of the macromers $[23,24]$.

Second, it has to be noted that the macromers were not purified after completion. These liquid macromers cannot be readily purified by precipitation in cold ethanol [13] and due to the low amounts of reagent used we chose not to remove access methacrylic anhydride and formed methacrylic acid via elaborate vacuum distillation. However, the presence of methacrylic acid can accelerate the hydrolytic degradation as carboxylic acids increase the hydrophilic nature of their surroundings leading to an increase in the water uptake [30]. Polymer batches for follow-up experiments (i.e. for investigating the drug release or longer degradation times) should, therefore, be purified to exclude the potential effect of the methacrylic acid.

Third, the $\mathrm{pH}$ of the PBS in the experiments was 7.4. However, the hydrolytic degradation of the anhydride-bond is base-catalyzed [31]. Therefore, degradation experiments in buffers with higher and lower pH should also be performed. For example, when considering drug delivery applications networks that degraded via hydrolytic anhydride-bond cleavage could potentially work as materials for orally administered colon drug-delivery as they can resist the acidic nature of the stomach.

The above-described results are encouraging as they show fast hydrolytically degradable PTMC networks can readily be prepared. However, it is also clear that for future applications several challenges remain. For drug delivery applications, polymer network degradation at more basic and acidic $\mathrm{pH}$ and the actual drug delivery characteristics remain to be investigated. Besides, the preparation and degradation of macromers of higher molecular weights and macromers with longer alkenyl chains can be considered. 

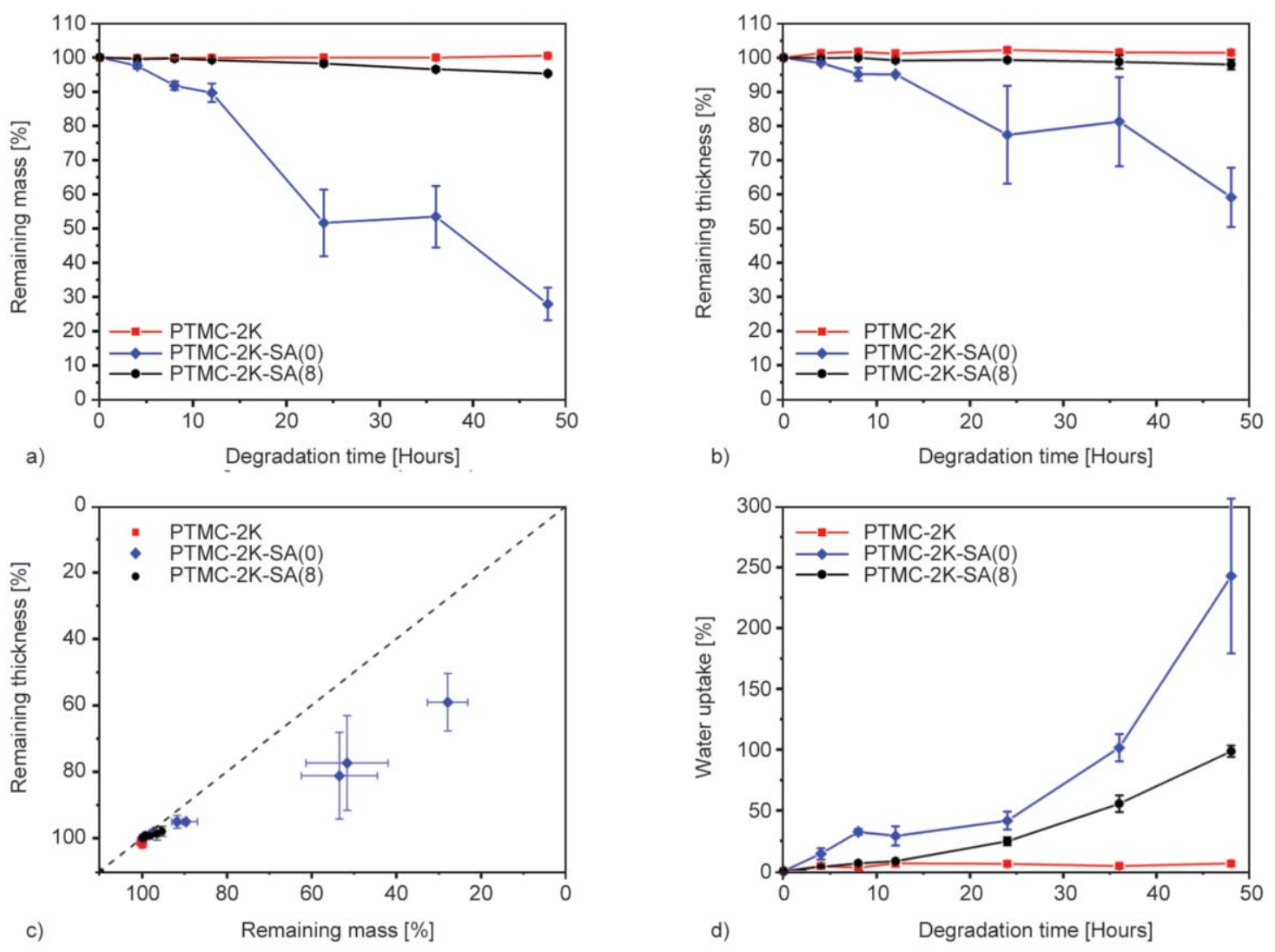

Figure 6. Degradation of PTMC-2K-anhydride networks. (a) Relative remaining mass as a function of time. (b) Relative remaining thickness as a function of time. (c) Relative remaining thickness as a function of relative remaining mass.

(d) Water update of the cylinders as a function of time.

For much higher molecular weights (i.e. $5000 \mathrm{~g} / \mathrm{mol}$ and higher) it has to be considered that the viscosity of the acid-functionalized oligomers prior methacrylation is much higher than the viscosities of the low molecular weight oligomers used in this study, even at elevated temperatures. The reaction conditions may need to be adjusted to achieve a proper degree of functionalization. In addition, these macromers will be much more viscous, which may require the use of solvent and solvent extraction in network preparation.

\section{Conclusions}

Photo-crosslinkable poly(trimethylene carbonate)anhydrides were readily synthesized by the acid-functionalization of PTMC oligomers with (alkenyl) succinic anhydrides and subsequent methacrylate functionalization. The obtained PTMC-anhydrides were viscous liquids that could be photo-crosslinked to obtain solid networks. These networks were shown to be fast-degrading due to hydrolysis, with the degradation mechanism showing characteristics of surface erosion.
The here presented results open up a wide field of potential applications for the PTMC anhydrides. However, investigating the behavior of the networks under more acidic and basic degradation conditions, as well as longer degradation times by varying the macromer molecular weight and/or the length of the alkenyl chains of the anhydride group is needed to obtain more control over the degradation.

\section{Acknowledgements}

This work made use of Aalto University Bioeconomy Facilities.

\section{References}

[1] van Bochove B., Grijpma D. W.: Photo-crosslinked synthetic biodegradable polymer networks for biomedical applications. Journal of Biomaterials Science, Polymer Edition, 30, 77-106 (2019). https://doi.org/10.1080/09205063.2018.1553105

[2] Pêgo A. P., Grijpma D. W., Feijen J.: Enhanced mechanical properties of 1,3-trimethylene carbonate polymers and networks. Polymer, 44, 6495-6504 (2003). https://doi.org/10.1016/S0032-3861(03)00668-2 
[3] Bat E., Harmsen M. C., Plantinga J. A., van Luyn M. J. A., Feijen J., Grijpma D. W.: Flexible scaffolds based on poly(trimethylene carbonate) networks for cardiac tissue engineering. Journal of Controlled Release, 148, e74-e76 (2010).

https://doi.org/10.1016/j.jconrel.2010.07.013

[4] Pêgo A. P., van Luyn M. J. A., Brouwer L. A., van Wachem P. B., Poot A. A., Grijpma D. W., Feijen J.: In vivo behavior of poly(1,3-trimethylene carbonate) and copolymers of 1,3-trimethylene carbonate with D,Llactide or $\varepsilon$-caprolactone: Degradation and tissue response. Journal of Biomedical Materials Research Part A, 67, 1044-1054 (2003).

https://doi.org/10.1002/jbm.a.10121

[5] Zhu K. J., Hendren R. W., Jensen K., Pitt C. G.: Synthesis, properties, and biodegradation of poly(1,3-trimethylene carbonate). Macromolecules, 24, 1736-1740 (1991).

https://doi.org/10.1021/ma00008a008

[6] Albertson A. C., Sjoling M.: Homopolymerization of 1,8dioxan-2-one to high molecular weight poly(trimethylene carbonate). Journal of Macromolecular Science Part A: Pure and Applied Chemistry, 29, 43-54 (1992). https://doi.org/10.1080/10101329208054106

[7] Albertsson A-C., Eklund M.: Influence of molecular structure on the degradation mechanism of degradable polymers: In vitro degradation of poly(trimethylene carbonate), poly(trimethylene carbonate-co-caprolactone), and poly(adipic anhydride). Journal of Applied Polymer Science, 57, 87-103 (1995).

https://doi.org/10.1002/app.1995.070570109

[8] Wang H., Dong J. H., Qiu K. Y., Gu Z. W.: Synthesis of poly(1,4-dioxan-2-one-co-trimethylene carbonate) for application in drug delivery systems. Journal of Polymer Science Part A: Polymer Chemistry, 36, 13011307 (1998).

https://doi.org/10.1002/(SICI)1099-

0518(199806)36:8<1301::AID-POLA13>3.0.CO;2-A

[9] Edlund U., Albertsson A-C., Singh S. K., Fogelberg I., Lundgren B. O.: Sterilization, storage stability and in vivo biocompatibility of poly(trimethylene carbonate)/ poly(adipic anhydride) blends. Biomaterials, 21, 945955 (2000).

https://doi.org/10.1016/S0142-9612(99)00268-9

[10] Zhang Y., Zhuo R-X.: Synthesis and drug release behavior of poly(trimethylene carbonate)-poly(ethylene glycol)-poly(trimethylene carbonate) nanoparticles. Biomaterials, 26, 2089-2094 (2005).

https://doi.org/10.1016/j.biomaterials.2004.06.004

[11] Sanson C., Schatz C., Le Meins J-F., Brûlet A., Soum A., Lecommandoux S.: Biocompatible and biodegradable poly(trimethylene carbonate)- $b$-poly(1-glutamic acid) polymersomes: Size control and stability. Langmuir, 26, 2751-2760 (2010).

https://doi.org/10.1021/la902786t
[12] Fukushima K.: Poly(trimethylene carbonate)-based polymers engineered for biodegradable functional biomaterials. Biomaterials Science, 4, 9-24 (2016).

https://doi.org/10.1039/C5BM00123D

[13] Schüller-Ravoo S., Feijen J., Grijpma D. W.: Flexible, elastic and tear-resistant networks prepared by photocrosslinking poly(trimethylene carbonate) macromers. Acta Biomaterialia, 8, 3576-3585 (2012).

https://doi.org/10.1016/j.actbio.2012.06.004

[14] van Bochove B., Hannink G., Buma P., Grijpma D. W.: Preparation of designed poly(trimethylene carbonate) meniscus implants by stereolithography: Challenges in stereolithography. Macromolecular Bioscience, 16, 1853-1863 (2016).

https://doi.org/10.1002/mabi.201600290

[15] Schüller-Ravoo S., Zant E., Feijen J., Grijpma D. W.: Preparation of a designed poly(trimethylene carbonate) microvascular network by stereolithography. Advanced Healthcare Materials, 3, 2004-2011 (2014).

https://doi.org/10.1002/adhm.201400363

[16] Geven M. A., Sprecher C., Guillaume O., Eglin D., Grijpma D. W.: Micro-porous composite scaffolds of photo-crosslinked poly(trimethylene carbonate) and nano-hydroxyapatite prepared by low-temperature extrusion-based additive manufacturing. Polymers for Advanced Technologies, 28, 1226-1232 (2017).

https://doi.org/10.1002/pat.3890

[17] Blanquer S. B. G., Gebraad A. W. H., Miettinen S., Poot A. A., Grijpma D. W., Haimi S. P.: Differentiation of adipose stem cells seeded towards annulus fibrosus cells on a designed poly(trimethylene carbonate) scaffold prepared by stereolithography. Journal of tissue engineering and regenerative medicine, 11, 2752-2762 (2017).

https://doi.org/10.1002/term.2170

[18] Chapanian R., Amsden B. G.: Combined and sequential delivery of bioactive VEGF165 and HGF from poly (trimethylene carbonate) based photo-cross-linked elastomers. Journal of Controlled Release, 143, 53-63 (2010).

https://doi.org/10.1016/j.jconrel.2009.11.025

[19] Rongen J. J., van Bochove B., Hannink G., Grijpma D. W., Buma P.: Degradation behavior of, and tissue response to photo-crosslinked poly(trimethylene carbonate) networks. Journal of Biomedical Materials Research Part A, 104, 2823-2832 (2016).

https://doi.org/10.1002/jbm.a.35826

[20] Helminen A. O., Korhonen H., Seppälä J. V.: Crosslinked poly(ester anhydride)s based on poly(E-caprolactone) and polylactide oligomers. Journal of Polymer Science Part A: Polymer Chemistry, 41, 3788-3797 (2003). https://doi.org/10.1002/pola.10951 
[21] Mönkäre J., Hakala R. A., Kovalainen M., Korhonen H., Herzig K-H., Seppälä J. V., Järvinen K.: Photocrosslinked poly(ester anhydride)s for peptide delivery: Effect of oligomer hydrophobicity on PYY3-36 delivery. European Journal of Pharmaceutics and Biopharmaceutics, 80, 33-38 (2012).

https://doi.org/10.1016/j.ejpb.2011.09.011

[22] Mönkäre J., Hakala R. A., Vlasova M. A., Huotari A., Kilpeläinen M., Kiviniemi A., Meretoja V., Herzig K. H., Korhonen H., Seppälä J. V., Järvinen K.: Biocompatible photocrosslinked poly(ester anhydride) based on functionalized poly( $\varepsilon$-caprolactone) prepolymer shows surface erosion controlled drug release in vitro and in vivo. Journal of Controlled Release, 146, 349-355 (2010). https://doi.org/10.1016/j.jconrel.2010.06.005

[23] Hakala R. A., Korhonen H., Meretoja V. V., Seppälä J. V.: Photo-cross-linked biodegradable poly(ester anhydride) networks prepared from alkenylsuccinic anhydride functionalized poly( $\varepsilon$-caprolactone) precursors. Biomacromolecules, 12, 2806-2814 (2011). https://doi.org/10.1021/bm200554c

[24] Hakala R. A., Korhonen H., Seppälä J. V.: Hydrolysis behaviour of crosslinked poly(ester anhydride) networks prepared from functionalised poly ( $\varepsilon$-caprolactone) precursors. Reactive and Functional Polymers, 73, 11-17 (2013).

https://doi.org/10.1016/j.reactfunctpolym.2012.10.002

[25] Gunatillake P. A., Adhikari R.: Biodegradable synthetic polymers for tissue engineering. European Cells and Materials, 5, 1-16 (2003).

https://doi.org/10.22203/eCM.v005a01
[26] Sun H., Mei L., Song C., Cui X., Wang P.: The in vivo degradation, absorption and excretion of PCL-based implant. Biomaterials, 27, 1735-1740 (2006). https://doi.org/10.1016/j.biomaterials.2005.09.019

[27] Asikainen S., van Bochove B., Seppälä J. V.: Drug-releasing biopolymeric structures manufactured via stereolithography. Biomedical Physics and Engineering Express, 5, 025008/1-025008/9 (2019).

https://doi.org/10.1088/2057-1976/aaf0e0

[28] Hakala R. A., Korhonen H., Holappa S., Seppälä J. V.: Hydrophobicities of poly( $\varepsilon$-caprolactone) oligomers functionalized with different succinic anhydrides. European Polymer Journal, 45, 557-564 (2009).

https://doi.org/10.1016/j.eurpolymj.2008.11.009

[29] Bat E., van Kooten T. G., Feijen J., Grijpma D. W.: Macrophage-mediated erosion of gamma irradiated poly(trimethylene carbonate) films. Biomaterials, 30, 3652-3661 (2009).

https://doi.org/10.1016/j.biomaterials.2009.03.033

[30] Rizwan M., Yahya R., Hassan A., Yar M., Azzahari A. D., Selvanathan V., Sonsudin F., Abouloula C. N.: pH sensitive hydrogels in drug delivery: Brief history, properties, swelling, and release mechanism, material selection and applications. Polymers, 9, 137/1-137/37 (2017). https://doi.org/10.3390/polym9040137

[31] Lopac S. K., Torres M. P., Wilson-Welder J. H., Wannemuehler M. J., Narasimhan B.: Effect of polymer chemistry and fabrication method on protein release and stability from polyanhydride microspheres. Journal of Biomedical Materials Research Part B: Applied Biomaterials, 91, 938-947 (2009).

https://doi.org/10.1002/jbm.b.31478 